\title{
Inhibition of Colony Stimulating Factor 1 Receptor Suppresses Neuroinflammation and Neonatal Hypoxic-Ischemic Brain Injury
}

\author{
Bohao Zhang ${ }^{1,2 t}$, Yunwei Ran ${ }^{3 t}$, Siting $W^{4}{ }^{4}$, Fang Zhang ${ }^{4}$, Huachen Huang ${ }^{4}$, \\ Changlian Zhu ${ }^{1,5}$, Shusheng Zhang ${ }^{2 *}$ and Xiaoan Zhang ${ }^{1 *}$
}

${ }^{1}$ Henan Key Laboratory of Child Brain Injury, Institute of Neuroscience and Third Affiliated Hospital of Zhengzhou University, Zhengzhou, China, ${ }^{2}$ Center of Advanced Analysis \& Gene Sequencing, Zhengzhou University, Zhengzhou, China, ${ }^{3}$ Medical Research Center, The Third Affiliated Hospital of Zhengzhou University, Zhengzhou, China, ${ }^{4}$ Department of Neurology, Tianjin Neurological Institute, Tianjin Medical University General Hospital, Tianjin, China, ${ }^{5}$ Department of Clinical Neuroscience, Center for Brain Repair and Rehabilitation, Institute of Neuroscience and Physiology, University of Gothenburg, Gothenburg, Sweden

OPEN ACCESS

Edited by:

Midori A. Yenari,

University of California, San Francisco,

United States

Reviewed by:

Tiina Kauppinen

University of Manitoba, Canada

Atsushi Mizuma

Tokai University, Japan

*Correspondence:

Shusheng Zhang

zsszz@126.com

Xiaoan Zhang

zxa@zzu.edu.cn

tThese authors have contributed equally to this work

Specialty section This article was submitted to

Stroke,

a section of the journal

Frontiers in Neurology

Received: 17 September 2020 Accepted: 28 January 2021 Published: 18 February 2021

Citation:

Zhang B, Ran Y, Wu S, Zhang F Huang $H$, Zhu $C$, Zhang $S$ and Zhang X (2021) Inhibition of Colony

Stimulating Factor 1 Receptor Suppresses Neuroinflammation and Neonatal Hypoxic-Ischemic Brain Injury. Front. Neurol. 12:607370. doi: 10.3389/fneur.2021.607370
Hypoxic-ischemic $(\mathrm{HI})$ brain injury is a major cause of neonatal death or lifetime disability without widely accepted effective pharmacological treatments. It has been shown that the survival of microglia requires colony-stimulating factor 1 receptor (CSF1R) signaling and microglia participate in neonatal $\mathrm{HI}$ brain injury. We therefore hypothesize that microglia depletion during a $\mathrm{HI}$ insult period could reduce immature brain injury. In this study, CD1 mouse pups were treated with a CSF1R inhibitor (PLX3397, 25 mg/kg/daily) or a vehicle from postnatal day 4 to day 11 (P4-11), and over $90 \%$ of total brain microglia were deleted at P9. Unilateral hemisphere HI injury was induced at P9 by permanently ligating the left common carotid arteries and exposing the pups to 10\% oxygen for 30 min to produce moderate left hemisphere injury. We found that the PLX3397 treatment reduced $\mathrm{HI}$ brain injury by $46.4 \%$, as evaluated by the percentage of brain infarction at $48 \mathrm{~h}$ after HI. Furthermore, CSF1R inhibition suppressed the infiltration of neutrophils (69.7\% reduction, $p=0.038)$, macrophages $(77.4 \%$ reduction, $p=0.009)$, and $T$ cells (72.9\% reduction, $p=0.008$ ) to the brain, the production of cytokines and chemokines (such as CCL12, CCL6, CCL21, CCL22, CCL19, IL7, CD14, and WISP-1), and reduced neuronal apoptosis as indicated by active caspase-3 labeled cells at $48 \mathrm{~h}$ after $\mathrm{HI}(615.20$ $\pm 156.84 / \mathrm{mm}^{2}$ vs. $\left.1,205.00 \pm 99.15 / \mathrm{mm}^{2}, p=0.013\right)$. Our results suggest that CSF1R inhibition suppresses neuroinflammation and neonatal brain injury after acute cerebral hypoxia-ischemia in neonatal mice.

Keywords: colony stimulating factor 1 receptor, PLX3397, microglia, neuroinflammation, neonatal hypoxic-ischemic brain injury

\section{INTRODUCTION}

Perinatal hypoxic-ischemic (HI) brain injury is a clinical syndrome associated with oxygen deprivation and is a major cause of neonatal death and long-term neurofunctional disabilities with limited therapies available (1-7). HI causes additional oxidative stress, excitotoxicity, and neuroinflammation, which further exacerbate the initial brain injury $(2,7)$. Neuroinflammation 
occurs as early as minutes after the insult and is a key contributor to acute perinatal HI brain injury $(8-11)$. Brain resident microglia play essential roles in immune surveillance of the brain microenvironment. After cerebral HI, brain microglia change their phenotype, promote the production of inflammatory factors, and recruit peripheral immune cells (12-14). The activated microglia change to the anti-inflammatory state and promote angiogenesis, tissue remodeling, and neurorepair at later stages after HI brain injury (14). However, the role of microglia in perinatal $\mathrm{HI}$ brain injury remains poorly understood (14).

Colony-stimulating factor 1 receptor (CSF1R) signaling is essential for microglia survival. Pexidartinib (PLX3397) is a CSF1R antagonist that has been reported to eliminate brain microglia temporarily and recoverably in adult mice (15-17). However, whether CSF1R inhibition can effectively eliminate microglia in the neonatal brain remains unknown. In this study, we determined the effects of CSF1R inhibition on microglia in the neonatal brain and cerebral HI injury using a mouse model.

\section{MATERIALS AND METHODS}

\section{Animals}

CD1 pregnant mice and their neonatal pups were used in this study. CD1 pregnant mice were purchased from Beijing Huafukang Biotechnology Co., Ltd. (Beijing, China). The mice were housed under controlled conditions, with free access to food and water, at $23 \pm 2{ }^{\circ} \mathrm{C}$, with $12 \mathrm{~h}$ light/dark periods. All the experiments were approved by the Committee on the Ethics of Animal Experiments of Tianjin Neurological Institute (Tianjin, China).

\section{Administration of PLX3397}

PLX3397 (Selleckchem, Houston, TX, USA) was dissolved in dimethyl sulfoxide as previously described, to a final concentration of $50 \mathrm{mg} / \mathrm{ml}(16,18)$. At postnatal day 4 (P4), both the male and female littermate pups were randomly divided into two groups. One group was orally administered PLX3397 (25 $\mathrm{mg} / \mathrm{kg}$ ) twice a day starting from $\mathrm{P} 4$ to the end of the experiment (P11), and the control group was given the same volume of vehicle. With this administration procedure of PLX3397, over $90 \%$ of total brain microglia were deleted at P9 or P11 in mice when tested by cell analysis with flow cytometry. Moreover, no serious side effects such as death or body weight loss were found in mice treated with PLX3397 in this study.

\section{Hypoxic-Ischemic Model}

P9 male and female CD1 mouse pups of 5-7 g bodyweight were used. A moderate cerebral HI brain injury were induced by permanent ligation of the left common carotid artery combined with exposure to $10 \%$ oxygen for $30 \mathrm{~min}$ as previously described $(19,20)$. In brief, mouse pups were anesthetized with isoflurane (3\% for induction and $1.5 \%$ for maintenance during surgery). A midline cervical incision was made in the anterior neck. The left common carotid artery was isolated and ligated with a sterile surgical line to occlude blood flow from P9 to P11. After surgery, the pups were placed back with the dams for $1 \mathrm{~h}$. The pups were then exposed to hypoxic conditions $\left(10 \% \mathrm{O}_{2}\right.$ and $90 \% \mathrm{~N}_{2}, 37^{\circ} \mathrm{C}$ ) for $30 \mathrm{~min}$. Following exposure, the pups were returned to the cages with the dams and were reared for another $48 \mathrm{~h}$ prior to assessment of brain injury and inflammation. This model is widely accepted as mimicking neonatal asphyxia and is known as the Rice-Vannucci model. The brain lesion can be found in the cortex, hippocampus, striatum, and thalamus, similar to the clinical finding in neonatal asphyxia (21). In this model, only the combination of unilateral permanent ligation and hypoxia can induce brain injury in the ipsilateral hemisphere. The hypoxia time needed adjustment to produce moderate brain injury because of the variable sensitivity to hypoxia of species, genetic background, and age of the mouse pups $(22,23)$. To maintain a consistent $\mathrm{HI}$ effect in mice, we strictly followed the experimental procedures and conditions in different batches of experiments. Each batch of $\mathrm{HI}$ induction was carried out in parallel for mice treated with PLX3397 and the vehicle.

\section{Corner Turn Test}

The corner-turning test was conducted to evaluate unilateral abnormalities of sensory and motor functions of HI mice. At $48 \mathrm{~h}$ after $\mathrm{HI}$ induction, mouse pups were put to a small opening side of a $30^{\circ}$ angle corner formed by two boards and encouraged to go into the corner. The mouse had to turn right or left to leave the corner. Each mouse repeated this procedure 10 times with an interval of $\geq 30 \mathrm{~s}$ between trials. The percentage of ipsilateral turns was then calculated as ipsilateral turns/(ipsilateral turn + contralateral turn) $\times 100$.

\section{2,3,5-Tripenyltetrazolium Chloride (TTC) Staining}

At $48 \mathrm{~h}$ after $\mathrm{HI}$, the pups were deeply anesthetized with isoflurane. The brain was immediately isolated after perfusion with $10 \mathrm{ml}$ ice-cold phosphate buffered saline (PBS) and stored at $-20^{\circ} \mathrm{C}$ for $20 \mathrm{~min}$ and subsequently was sliced into $1.5 \mathrm{~mm}$ coronal sections. The sections were immersed in $2 \%$ TTC solution (Sigma-Aldrich, USA) at $37^{\circ} \mathrm{C}$ for $20 \mathrm{~min}$ in the dark. After washing with distilled $\mathrm{H}_{2} \mathrm{O}$, the sections were fixed in $4 \%$ paraformaldehyde for $20 \mathrm{~min}$ and photographed. The infarcted portions were identified as the white areas without TTC staining. The infarcted areas of each section were traced and measured using Image-Pro Plus 6.0 (U.S. National Institutes of Health, Washington, DC) (24). The total infarct volume was calculated by summation of the representative infarct volume of each section. The infarction percentage was calculated as follows: ([total contralateral hemispheric volume] - [total ipsilateral hemispheric stained volume]) / (total contralateral hemispheric volume $\times 2) \times 100 \%(19,25)$.

\section{Flow Cytometry}

Flow cytometry was performed to analyze the number of microglia and infiltration of immune cells in the brain. The pups were anesthetized and perfused intracardially with ice-cold $0.1 \mathrm{M}$ PBS ( $\mathrm{pH}$ 7.4). The brain was then isolated and rapidly placed in ice-cold PBS. The olfactory bulb, cerebellum, and brain stem were discarded, and only the cerebral hemispheres were retained. The cerebral tissue was cut with scissors and mechanically 
passed through a $40 \mu \mathrm{m}$ filter to obtain a cell suspension. To remove the myelin sheath from the cell suspension, $10 \mathrm{ml}$ of $30 \%$ Percoll (Sigma, St. Louis, MO) was added to the brain tissue suspension, followed by density gradient centrifugation at $4^{\circ} \mathrm{C}, 600 \mathrm{~g}$ for $20 \mathrm{~min}$. The isolated cell suspensions were stained with fluorescent-dye conjugated anti-mouse antibodies: CD45 (103108, BioLegend, San Diego, CA), CD11b (101216, BioLegend, San Diego, CA), Ly6G (127608, BioLegend, San Diego, CA), F4/80 (123116, BioLegend, San Diego, CA), CD3 (100327, BioLegend, San Diego, CA), CD19 (115519, BioLegend, San Diego, CA), and CSF1R (135510, BioLegend, San Diego, CA, USA). A FACS Aria III flow cytometer (BD Biosciences, San Jose, CA) was used to acquire flow cytometric data. Data were analyzed using Flow Jo software, version 10.0.7 (Informer Technologies, Walnut, CA).

\section{Immunofluorescence Staining}

The animals were anesthetized and perfused intracardially with ice-cold 0.1M PBS ( $\mathrm{pH} 7.4$ ) followed by $4 \%$ paraformaldehyde. The cerebrums were post-fixed in $4 \%$ paraformaldehyde for $48 \mathrm{~h}$, embedded in paraffin, and cut into $5 \mu \mathrm{m}$ coronal sections. The paraffin-embedded sections were heated at $95^{\circ} \mathrm{C}$ in $0.01 \mathrm{M}$ sodium citrate buffer solution $(\mathrm{pH} 6.0$ ) for $30 \mathrm{~min}$ to repair the antigen. The sections were blocked in $5 \%$ bovine serum albumin in PBS containing $0.2 \%$ Triton at $25^{\circ} \mathrm{C}$ for $2 \mathrm{~h}$, and then incubated with mouse anti-mouse NeuN (ab104224, Abcam, 1:1000 dilution) and rabbit anti-mouse caspase-3 (Cell Signaling Technology, 9661S; 1:1000) as primary antibodies at $4^{\circ} \mathrm{C}$ overnight. After washing thrice with PBS, the sections were incubated with biotinylated goat anti-mouse 488 (A11001, Invitrogen, Carlsbad, CA, 1:500 dilution) and goat antirabbit 546 (A11010, Invitrogen, Carlsbad, CA, 1:500 dilution) secondary antibodies at room temperature for $1 \mathrm{~h}$. The stained sections were imaged with a fluorescence microscope (Model BX61, Olympus, Center Valley, PA, USA). Sections without antibody staining were used as negative controls.

\section{Real-Time PCR}

Total RNA was isolated using TRIzol reagent (Thermo Fisher Scientific). Analysis was performed in a thermocycler (7500, Applied Biosystems, USA) using the following conditions: initial denaturation for $10 \mathrm{~s}$ at $95^{\circ} \mathrm{C}, 40$ cycles of $5 \mathrm{~s}$ denaturation at $95^{\circ} \mathrm{C}, 30 \mathrm{~s}$ annealing at $60^{\circ} \mathrm{C}$, and $1 \mathrm{~min}$ extension at $95^{\circ} \mathrm{C}$. The following primers were used for analysis: CCL6 forward GGCTT TCAAGACACTTCTTCAG, CCL6 reverse CCCTCCTGCTGAT AAAGATGAT; CCL12 forward CCAGTCACGTGCTGTTATA ATG, CCL12 reverse AGACGTCTTATCCAAGTGGTTT; CD14 forward CAAGTTCCCGACCCTCCAAG, CD14 reverse GCA-T CCCGCAGTGAATTGTG; CX3CL1 forward GAGGCCACCCT AGACCACTA, CX3CL1 reverse CACTGGAGTTGGGGTGTC AA; M-CSF forward TGATTGGGAATGGACACCTG, M-CSF reverse AAAGGCAATCTGGCATGAAGT; IL-7 forward TCT GCTGCCTGTCACATCATCT, IL-7 reverse AAGTTTGGTTC ATTATTCGGG; ICAM-1 forward TGTCAGCCACCATGCCT TAG, ICAM-1 reverse CAGCTTGCACGACCCTTCTA; VEGF forward GCCAGACAGGGTTGCCATAC, VEGF reverse GGA GTGGGATGGATGATGTCAG; WISP-1 forward AACTGCAT
AGCCTACACTAGTC, WISP-1 reverse ATTGACGTTAGAGA TCCGAGTG; and $\beta$-actin forward CTACCTCATGAAGATC CTGACC, $\beta$-actin reverse CACAGCTTCTCTTTGATGTCAC. The relative expression levels were calculated using the $2^{-\Delta \Delta C t}$ method and normalized with $\beta$-actin as the internal reference.

\section{Cytokine Array}

Cytokines in the cerebral tissue lysates were measured using a mouse XL cytokine array kit (ARY028, R\&D Systems). Briefly, cerebral tissue was removed and homogenized in PBS with protease inhibitors, aprotinin (A6279, Sigma), leupeptin (1167, Tocris), and pepstatin (1190, Tocris). After homogenization, Triton X-100 was added to the samples to a final concentration of $1 \%$. Protein homogenates were centrifuged at $10,000 \times \mathrm{g}$ for $5 \mathrm{~min}$ to remove cellular debris and stored at $-80^{\circ} \mathrm{C}$ until assay. The protein levels of cytokines were detected by exposure of the membrane to the X-ray film. The Gel-Pro analyzer was used to evaluate the intensity of the developed dots.

\section{Statistical Analysis}

All statistical data are presented as mean \pm SEM. Experimental and control group allocations, data collection, and data analysis were blinded by using different investigators or masking sample labels. Animals were randomly assigned to experimental groups. All experiments were successfully reproduced at least three times. A two-tailed unpaired Student's $t$-test was used to compare the two groups. One-way ANOVA was used for comparison between two groups. Two-way ANOVA with multiple comparisons was used to compare multigroup data. A level of $p<0.05$ was considered significant. Statistical analyses were performed using the Prism 6.0 software (GraphPad).

\section{RESULTS}

\section{PLX3397 Reduces Brain Microglia Numbers and Brain Injury in Neonatal Mice}

To deplete brain microglia, neonatal mice were treated with PLX3397 (25 mg/kg body weight) or vehicle at P4 prior to HI induction (Figure 1A). We found that brain microglia expressed CSF1R in neonatal mice (Figure 1B), and oral administration of PLX3397 for five consecutive days can effectively reduce 90\% of microglia in the brain of normal neonatal mice (Figure 1C).

Induction of $\mathrm{HI}$ in neonatal CD1 mice was performed at P9. We then evaluated HI-induced brain lesion size using TTC staining. Compared to HI mice treated with the vehicle, brain infarct size was significantly decreased in the mice treated with PLX3397 at $48 \mathrm{~h}$ after HI (Figure 2A). The relative infarct size was $28.70 \pm 1.66 \%$ in $\mathrm{HI}$ mice treated with the vehicle and 15.37 $\pm 3.17 \%$ in HI mice treated with PLX3397, which accounted for a $46.4 \%$ reduction of brain injury $(p=0.001)$ (Figure $2 B$ ). We further analyzed the infarction by gender and the relative infarct size was $27.3 \pm 3.4 \%$ in males and $29.8 \pm 1.4 \%$ in females in the vehicle treated group ( $p=0.105$, data not shown), and $15.3 \pm 4.9 \%$ in males and $15.4 \pm 0.4 \%$ in females in the PLX3397 treated group ( $p=0.99$, data not shown). These results indicate that inhibition of CSF1R prevents brain injury in 
A
PLX3397 or vehicle (25mg/kg per day)
Brain hypoxic-
$>$ Brain TTC staining
(25 ischemia induction
$>$ Brain immune profile assay
$>$ Brain cytokine expression array

Day 4

Day 9

Day 11

Days after birth

B

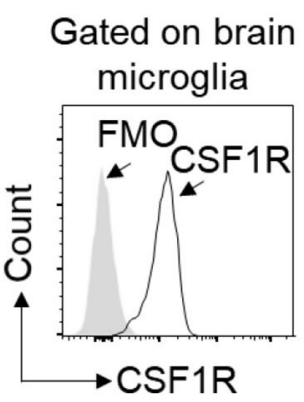

C
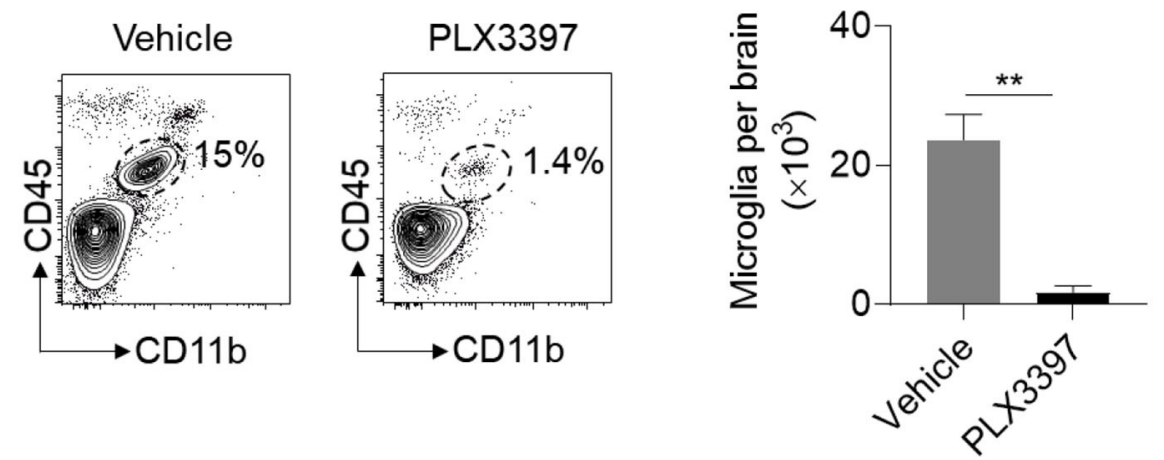

FIGURE 1 | PLX3397 reduces brain microglia in neonatal CD1 mice. (A) Experimental schematic design of PLX3397 administration in neonatal CD1 mice. (B) Expression of CSF1R on brain microglia (CD45 int $C D 11 b^{+}$cells) of neonatal CD1 mice at 4 days after birth. FMO stands for Fluorescence Minus One Control. (C) Flow cytometry plots and statistics of brain microglia $\left(\mathrm{CD} 11 \mathrm{~b}^{+} \mathrm{CD} 45^{\text {int }}\right.$ cells) at 9 days after birth in neonatal CD1 mice treated with PLX3397 ( $n=6,3$ males and 3 females) or the vehicle ( $n=6,3$ males and 3 females). Unpaired two-tailed $t$-test. Data are presented as mean \pm SEM, ${ }^{* *} p<0.01$.

A

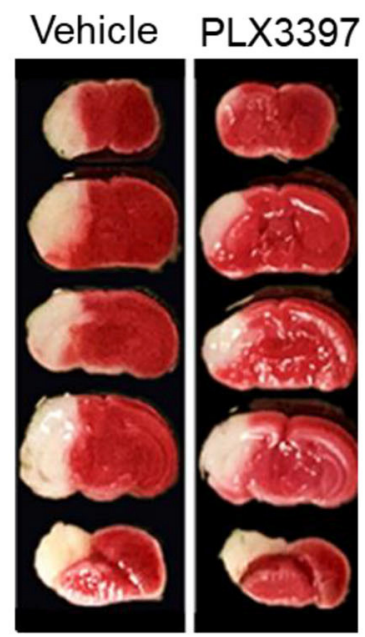

B

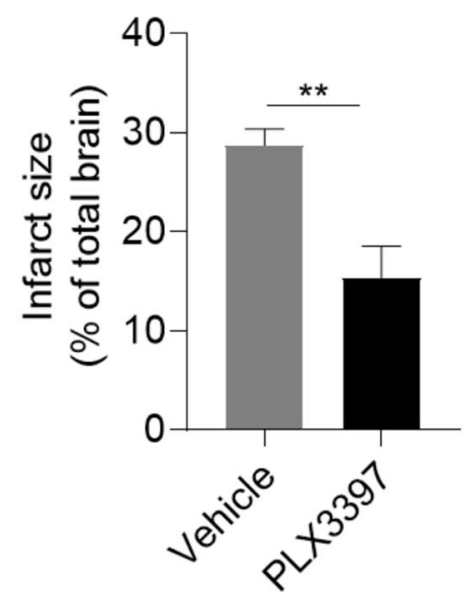

c

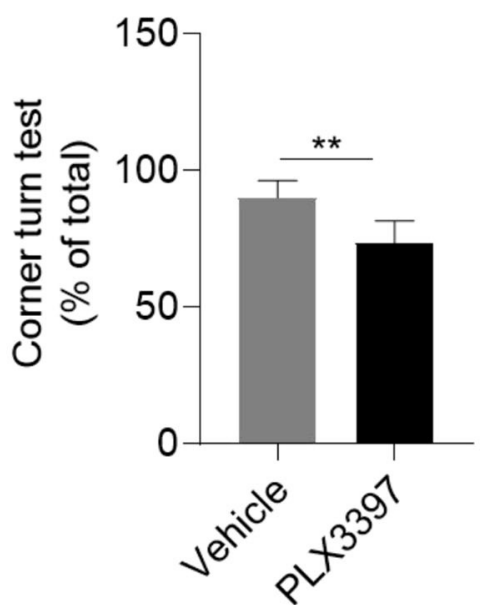

FIGURE 2 | PLX3397 attenuates neonatal hypoxic-ischemic brain injury in mice. (A) 2,3,5-Tripenyltetrazolium chloride (TTC) staining shows brain injury at 48 h after hypoxic-ischemic brain injury induction in neonatal CD1 mice treated with PLX3397 or the vehicle. (B) Statistics of infarct size measured by $T$ TC staining in HI mice treated with PLX3397 ( $n=9,6$ males and 3 females) or vehicle $(n=11,5$ males and 6 females) Unpaired two-tailed $t$-test. (C) Corner turn test at $48 \mathrm{~h}$ after $\mathrm{HI}$ induction in mice treated with PLX3397 ( $n=6,3$ males and 3 females) or vehicle ( $n=6,3$ males and 3 females). Unpaired two-tailed $t$-test. Data are presented as mean \pm SEM, ${ }^{* *} p<0.01$.

neonatal HI mice and there was no gender difference in brain injury in either vehicle of PLX3397 treated mice at $48 \mathrm{~h}$ after $\mathrm{HI}$ induction.
As unilateral visible lesions were found after $\mathrm{HI}$ induction (Figure 2A), we further used the corner turn test to analyze unilateral disability in motor and sensory function of $\mathrm{HI}$ mice 
A

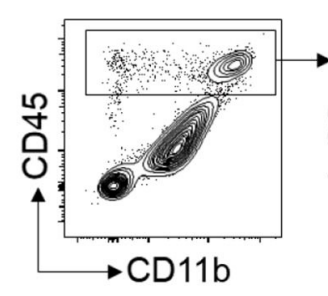

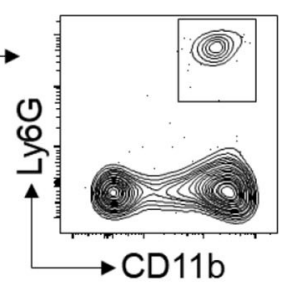

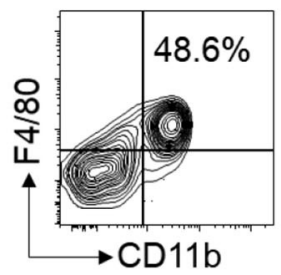

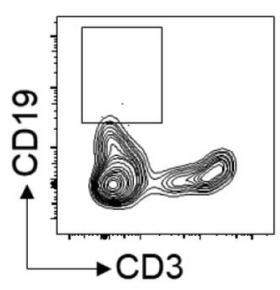

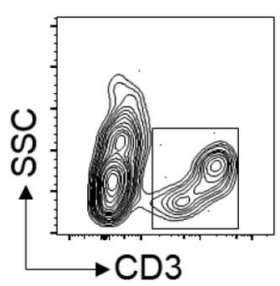

B

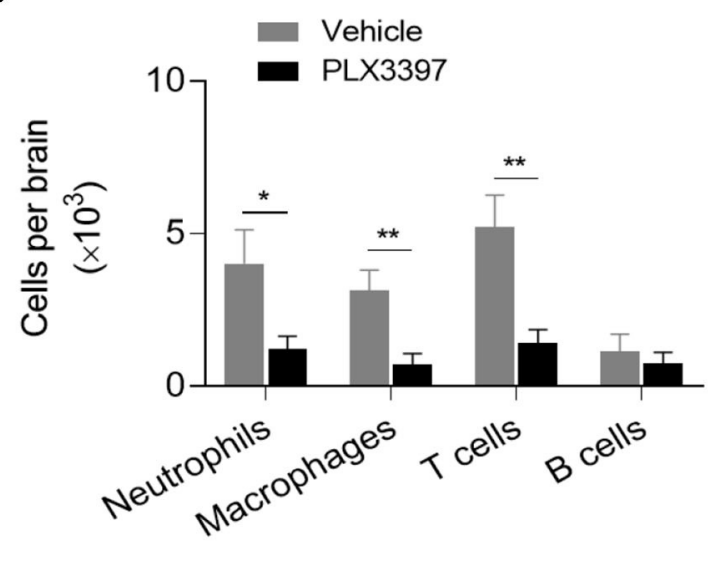

FIGURE 3 | PLX3397 reduces immune cell recruitment into the brains of HI mice. (A) Flow cytometry gating strategy for neutrophils $\left(\mathrm{CD} 45^{\text {high }}\right.$ CD11 ${ }^{+}$Ly6G $\left.{ }^{+}\right)$, macrophages $\left(\mathrm{CD} 45^{\text {high }} \mathrm{CD} 11 \mathrm{~b}^{+} \mathrm{F} 4 / 80^{+}\right)$, T cells $\left(\mathrm{CD} 45^{\text {high }} \mathrm{CD} 3^{+}\right)$, and B cells $\left(\mathrm{CD} 45^{\text {high }} \mathrm{CD} 3^{-} \mathrm{CD} 19^{+}\right)$in the brains of neonatal HI mice. (B) The quantitation of brain neutrophils, macrophages, T cells, and B cells at $48 \mathrm{~h}$ after $\mathrm{HI}$ in mice treated with PLX3397 ( $n=6,4$ males and 2 females) or the vehicle $(n=6,4$ males and 2 females). Unpaired two-tailed $t$-test. Data are presented as mean $\pm \mathrm{SEM},{ }^{*} p<0.05,{ }^{* \star} p<0.01$.

as previous published (26). At $48 \mathrm{~h}$ after HI induction, our data indicated that HI mice treated with PLX3397 had less unilateral asymmetry than $\mathrm{HI}$ mice treated with the vehicle ( $90 \pm 2.6 \%$ vs. $73.4 \pm 3.3 \%, p=0.0087$ ) (Figure 2C).

\section{CSF1R Inhibition Reduces the Recruitment of Circulating Immune Cells Into the Brain}

In addition to microglia, brain-recruited neutrophils, macrophages, and lymphocytes also contribute to brain injury in HI mice. Thus, we measured whether PLX3397 affects brain infiltration of peripheral immune cells in neonatal HI mice. At $48 \mathrm{~h}$ after $\mathrm{HI}$ induction, our data indicated that the number of neutrophils, macrophages, and T cells in the whole brain tissue was much lower in mice treated with PLX3397 (Figure 3). The number of neutrophils, macrophages, and T cells were $4.02 \pm$ $1.10 \times 10^{3}, 3.14 \pm 0.66 \times 10^{3}$, and $5.21 \pm 1.06 \times 10^{3}$ in HI mice treated with the vehicle and $1.22 \pm 0.40 \times 10^{3}, 0.71 \pm 0.36 \times 10^{3}$, and $1.41 \pm 0.45 \times 10^{3}$ in HI mice treated with PLX3397, which accounted for a $69.7,77.4$, and $72.9 \%$ reduction of infiltration of neutrophils $(p=0.038)$, macrophages $(p=0.009)$, and T cells $(p=0.008)$ to the brain. This result suggests that inhibition of CSF1R by PLX3397 attenuates neuroinflammation in HI mice.

\section{CSF1R Inhibition Alters Cytokine/Chemokine Profile in the Mouse Brain After HI Injury}

Chemokines and cytokines are important soluble factors in regulating the immune response triggered by tissue injury. As PLX3397 treatment reduced immune cells in injured brain tissue of HI mice (Figure 3), we wondered whether there is any difference in the cytokine/chemokine expressing profile between HI mice treated with PLX3397 and the vehicle. We used a proteome profiler mouse XL cytokine array to assess the expression of hundreds of cytokines/chemokines in the brain lysate of HI mice treated with PLX3397 or the vehicle. In Figure 4A, we show the 18 differently expressed factors at protein level between HI mice treated with PLX3397 or the vehicle ( $p$ $<0.05)$. In these differently expressed factors except M-CSF and DPPIV, their protein levels were reduced in the brains of HI mice treated with PLX3397. Fifteen of these 18 factors were related to chemotaxis and immune response (e.g., CCL6, CCL12, CCL19, CCL21 CCL22, CXCL16, CD14, IL-7, M-CSF, and myeloperoxidase) or the regulation of blood-brain barrier integrity (MMP-2, MMP3, ICAM-1, VEGF, and WISP-1).

We further analyzed the brain mRNA levels of some representative differently expressed factors related to regulation of chemotaxis (CCL2, CCL12), immune response (CD14, MCSF, IL-7), and blood brain barrier (BBB) integrity (ICAM-1, VEGF, WISP-1). However, our data indicated that not all these representative factors had synergistic changes in both mRNA and protein level in the brain of HI mice treated with PLX3397 (e.g., IL-7, ICAM-1, VEGF, and WISP-1) (Figure 4B). CX3CL1 was used as a negative control that is not significantly changed at protein level in the brain by cytokine array (data not shown), and we also did not find a difference in mRNA level of brain CX3CL1 between HI mice treated with PLX3397 and the vehicle (Figure 4B). These results demonstrated that PLX3397 alters the transcription or (and) translation of some factors in regulating immune cells' mobilization and BBB integrity in the brain after HI injury.

\section{CSF1R Inhibition Attenuates Neuronal Death in Injured Brain Tissue in HI Mice}

To determine the effects of PLX3397 on HI-induced neuronal death, we quantified the numbers of $\mathrm{NueN}^{+}$cells and active caspase $3^{+}$cells in the lesion area of $\mathrm{HI}$ mice at $48 \mathrm{~h}$ after $\mathrm{HI}$ induction. Compared to mice treated with the vehicle, the PLX3397 treatment preserved more neurons $(6,423.60 \pm 681.18$ 
A

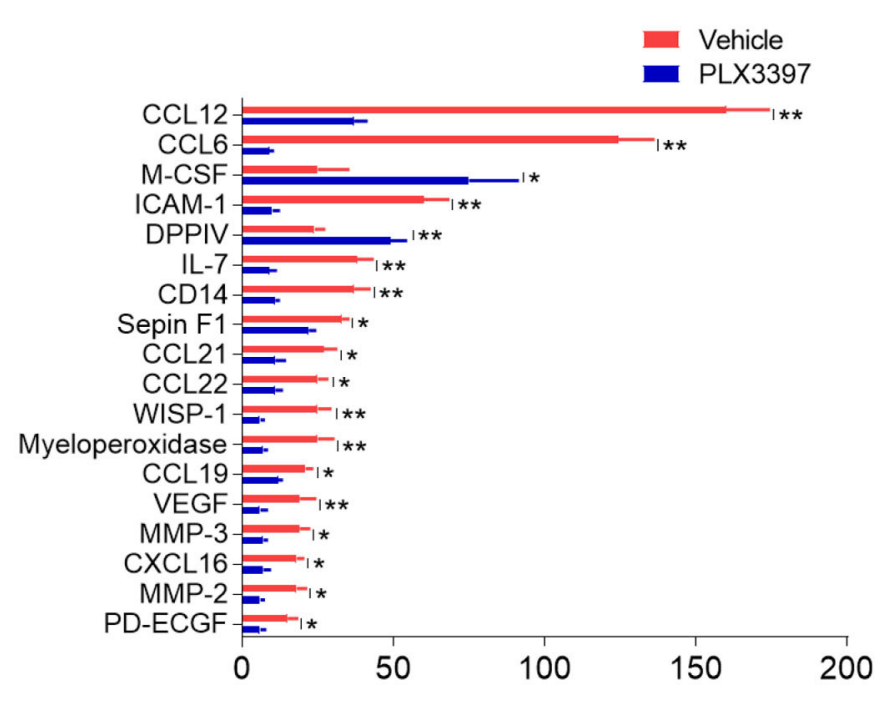

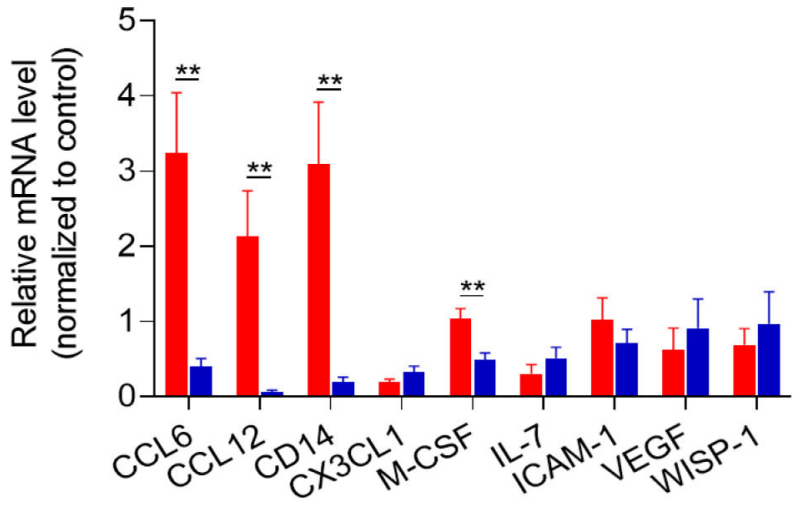

FIGURE 4 | PLX3397 changes brain cytokine/chemokine profile in HI mice. (A) The total differentially expressed cytokines/chemokines in the brains of HI mice treated with PLX3397 or the vehicle. Brain tissues were collected at $48 \mathrm{~h}$ after HI induction and the protein levels were measured with a cytokine/chemokine profile ELISA array kit in which 111 factors were detected. $n=3$ independent experiments. Each independent experiment needed 10 mice per group (5 males and 5 females). Unpaired two-tailed $t$-test. (B) The mRNA levels of representative differentially expressed factors related to regulation of chemotaxis (CCL2, CCL12), immune response (CD14, M-CSF, IL-7), and BBB integrity (ICAM-1, VEGF, and WISP-1). CX3CL1 was as a negative control that was not significantly changed in protein level between $\mathrm{HI}$ mice treated with PLX3397 or the vehicle (data not shown). Brain tissues were collected at $48 \mathrm{~h}$ after $\mathrm{HI}$ from $\mathrm{HI}$ mice treated with PLX3397 or the vehicle. $n=5$ mice per group. Unpaired two-tailed $t$-test. Data are presented as mean $\pm \mathrm{SEM},{ }^{\star} p<0.05,{ }^{* \star} p<0.01$.

vs. $3,790.40 \pm 656.80, p=0.024$ ) in injured brain tissue (around the infarct in the cortex) of HI mice, accompanied by a reduction in cell apoptosis $(615.20 \pm 156.84$ vs. $1,205.00 \pm 99.15, p=$ 0.013) (Figure 5). These results indicate that inhibition of CSF1R by PLX3397 reduces cellular apoptosis in injured brain tissue in HI mice.

\section{DISCUSSION}

In the present study, we showed that CSF1R inhibition reduces brain injury in neonatal mice after $\mathrm{HI}$ insult. As documented here, most microglia can be effectively eliminated by CSF1R inhibition in neonatal mice. In contrast to adult mice that require 2-3 weeks of $50 \mathrm{mg} / \mathrm{kg}$ PLX3397 treatment to eliminate $90 \%$ of brain microglia (15), we found that a lower dosage of PLX3397 $(25 \mathrm{mg} / \mathrm{kg}$ ) for 5 days can effectively reduce $\sim 90 \%$ of microglia in the neonatal mouse brain. Importantly, the reduced neuronal death and infarct size suggest that targeting CSF1R could be a promising approach to limiting $\mathrm{HI}$ injury in the neonatal brain.

Our study provides new evidence that microglia depletion reduces brain ischemia injury in mice, which is opposite to some previous studies with brain ischemic injury models (15, 27). This discrepancy could be related to age, gender, strain of animals, and the type of models used (27-31). With different strains, age, and gender of mice, there are controversial results on whether microglia are protective or harmful to ischemic brain injury $(14,15,32-35)$. For example, microglia depletion with PLX3397 provided neuroprotective effects by an inhibitory action on the astrocyte response in adult transient middle cerebral artery occlusion mice (15). However, the role of astrocytes in the development of ischemic brain lesions is unclear in neonates (14). Meanwhile, neonatal and adult microglia have a distinguishing immune response and cytokine/chemokine production profile after encountering brain ischemia. For example, adult microglia are more sensitive to the stimulation of Toll-like receptors ligands and produce higher matrix metalloproteinases, while the ability to respond to nitric oxide is markedly enhanced in neonatal microglia $(34,36)$. Thus, it is possible that glia cells have different effects on acute brain ischemic lesions in neonatal and adult mice due to their different immune response abilities.

Apart from mouse age, the difference in mouse gender and genetic background may also affect the outcome of HI mice. For example, there is a gender difference in HI-induced acute brain injury in C57BL6 mice (26), as female C57BL6 mice have smaller brain lesions than male mice after HI induction. It is thought that different apoptotic cell death mechanisms are activated in neonatal male and female brains after $\mathrm{HI}$ insult (37). A recent tamoxifen-induced conditional genetic microglia depletion study by also showed aggravated brain injury after cerebral HI in neonatal mice and more predominantly in males (27). In the current study, we did not find a gender difference in brain lesion at $48 \mathrm{~h}$ after $\mathrm{HI}$ in mice treated with PLX3397 or the vehicle. One reason is that gender differences may not be statistically significant at $48 \mathrm{~h}$ after $\mathrm{HI}$, as a previous study indicated that female HI mice have smaller brain lesions than male mice at $72 \mathrm{~h}$ but not earlier than $24 \mathrm{~h}$ after HI induction (26). Another reason is that we used CD1 mice in this study and this strain is more susceptible to HI injury than C57BL6 mouse pups (22). The 
A

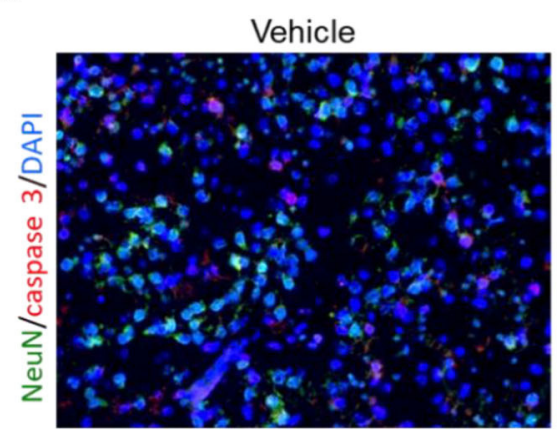

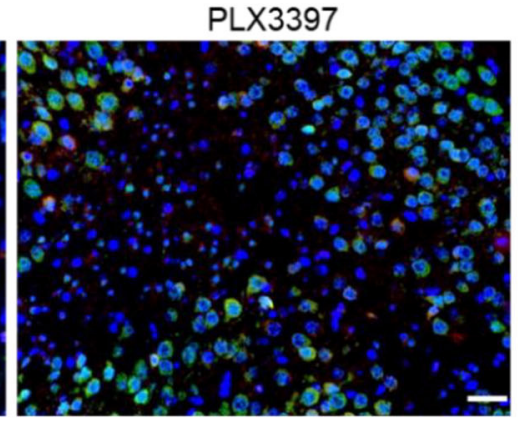

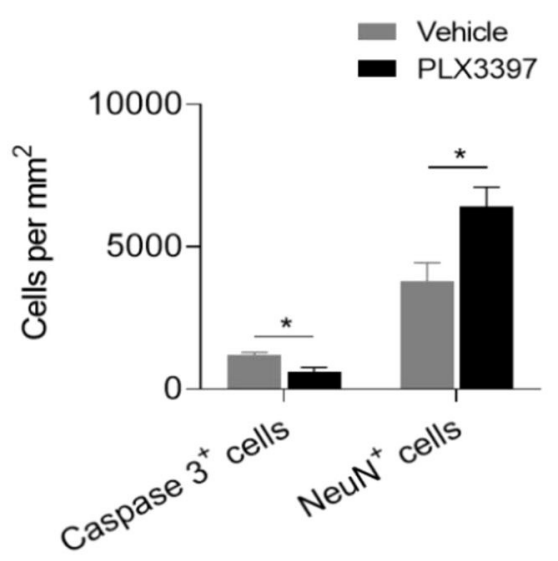

FIGURE 5 | PLX3397 attenuates cell death in injured brain tissue of HI mice. (A) immunostaining of activated caspase 3 (red) and neuron (green) in injured brain tissue at $48 \mathrm{~h}$ after $\mathrm{HI}$ induction in mice treated with PLX3397 or the vehicle. Scale bar $=20 \mu \mathrm{m}$. (B) Quantification of neuron and cell apoptosis at $48 \mathrm{~h}$ after $\mathrm{HI}$ induction in injured brain tissue of $\mathrm{HI}$ mice treated with PLX3397 ( $n=6,1$ male and 5 females) or vehicle $(n=6,3$ males and 3 females). Unpaired two-tailed $t$-test. Data are presented as mean \pm SEM, ${ }^{*} p<0.05$.

difference in genetic background may affect the brain pathology development including microglia activity and neural function after HI, resulting in different brain injury mechanisms and outcomes. In addition, not all analysis had equal sex distribution in our results, such as the brain lesions and the cell death between mice treated with PLX3397 and vehicle as shown in Figures 2B, 5. Thus, the potential effects of sex difference on $\mathrm{HI}$ induced brain lesion and cell death between mice treated with PLX3397 and vehicle needs to be further studied. In our further studies, we will pay attention to whether the gender, mouse strain, and even HI model procedure affect microglia activity and HI outcomes by PLX3397 in neonatal mice.

In this study, PLX3397 altered the immune profile in the brain of $\mathrm{HI}$ mice, manifested in the decrease of microglia, brain infiltrated immune cells, and chemokine/cytokine expression in brain tissue. Although we did not study the downstream molecular mechanism of PLX3397 on brain lesion and neuron death, microglia related neuroinflammation may be a potential mechanism. Studies suggest that microglia are rapidly activated by acute brain injuries including brain ischemia and contribute to acute brain injury and chronic brain repair (38). As for HI, microglia exhibit different functional phenotypes during injury and brain repair, similar to $\mathrm{M} 1, \mathrm{M} 2 \mathrm{~A}$, and $\mathrm{M} 2 \mathrm{~b}$ phenotypes proposed in other models $(12,39)$. Microglia mainly differentiate into the M1 phenotype and are thought to play a detrimental role in brain injury after $\mathrm{HI}$, at least in the acute stage (40-43), though the mechanism is not fully understood. Thus, the effects of PLX3397 on brain lesion and neuron death may be related to inhibited M1 polarization and brain inflammation in this study. Furthermore, our cytokine/chemokine array result provides additional cues of the mechanism on attenuated brain injury in HI + PLX3397 mice. Among the 18 differently expressed cytokines/ chemokines, some of them may be directly harmful to neurons. For example, a recent study showed that CCL6 can aggravate hypoxia-reoxygenation-induced cell apoptosis (44). In our study, we found that protein and mRNA levels of CCL6 were significantly reduced in whole brain tissue of HI mice treated with PLX3397. This reduced CCL6 level may be related to neuron protection in HI mice treated with PLX3397. Another example is M-CSF. It is reported that astrocytes constitutively express M-CSF that increases phagocytosis and proliferation of microglia $(45,46)$. M-CSF also promotes the differentiation of monocytes into M2 macrophages with an "anti-inflammatory" cytokine repertoire and functions $(47,48)$. It is interesting that mice treated with PLX3397 had a higher protein level of MCSF after HI induction in our study. This high level of M-CSF may facilitate the clearance of dead cells and subsequently brain repair. Thus, it is worth dissecting the potential mechanism of PLX3397 on brain injury in HI mice in future studies.

Overall, this study demonstrates that inhibition of CSF1R suppresses neuroinflammation and HI injury in the neonatal brain.

\section{DATA AVAILABILITY STATEMENT}

The raw data supporting the conclusions of this article will be made available by the authors, without undue reservation.

\section{ETHICS STATEMENT}

The animal study was reviewed and approved by the Committee on the Ethics of Animal Experiments of Tianjin Neurological Institute (Tianjin, China).

\section{AUTHOR CONTRIBUTIONS}

$\mathrm{XZ}, \mathrm{SZ}$, and $\mathrm{CZ}$ formulated the study concept and designed research. BZ, YR, SW, FZ, and $\mathrm{HH}$ performed experiments. BZ, YR, and SW analyzed the data, interpreted the results, and wrote the paper. All authors contributed to the article and approved the submitted version. 


\section{FUNDING}

This study was supported in part by National Science Foundation of China (Grants 80870983); International Cooperation Project of Henan Provincial Science and Technology Department (Grants 172102410001);

\section{REFERENCES}

1. Zhang S, Li B, Zhang X, Zhu C, Wang X. Birth asphyxia is associated with increased risk of cerebral palsy: a meta-analysis. Front Neurol. (2020) 11:704. doi: 10.3389/fneur.2020.00704

2. Yildiz EP, Ekici B, Tatli B. Neonatal hypoxic ischemic encephalopathy: an update on disease pathogenesis and treatment. Expert Rev Neurother. (2017) 17:449-59. doi: 10.1080/14737175.2017.1259567

3. Dixon BJ, Reis C, Ho WM, Tang J, Zhang JH. Neuroprotective strategies after neonatal hypoxic ischemic encephalopathy. Int J Mol Sci. (2015) 16:22368401. doi: 10.3390/ijms160922368

4. Tagin M, Zhu C, Gunn AJ. Beneficence and nonmaleficence in treating neonatal hypoxic-ischemic brain injury. Dev Neurosci. (2015) 37:30510. doi: $10.1159 / 000371722$

5. Douglas-Escobar M, Weiss MD. Hypoxic-ischemic encephalopathy: a review for the clinician. JAMA Pediatr. (2015) 169:397403. doi: 10.1001/jamapediatrics.2014.3269

6. Li T, Xu F, Cheng X, Guo X, Ji L, Zhang Z, et al. Systemic hypothermia induced within 10 hours after birth improved neurological outcome in newborns with hypoxic-ischemic encephalopathy. Hosp Pract. (2009) 37:14752. doi: 10.3810/hp.2009.12.269

7. Inder TE, Volpe JJ. Mechanisms of perinatal brain injury. Semin Neonatol. (2000) 5:3-16. doi: 10.1053/siny.1999.0112

8. Liu F, McCullough LD. Inflammatory responses in hypoxic ischemic encephalopathy. Acta Pharmacol Sin. (2013) 34:112130. doi: 10.1038/aps.2013.89

9. Lai JCY, Rocha-Ferreira E, Ek CJ, Wang X, Hagberg H, Mallard C. Immune responses in perinatal brain injury. Brain Behav Immun. (2017) 63:21023. doi: 10.1016/j.bbi.2016.10.022

10. Li B, Concepcion K, Meng X, Zhang L. Brain-immune interactions in perinatal hypoxic-ischemic brain injury. Prog Neurobiol. (2017) 159:5068. doi: 10.1016/j.pneurobio.2017.10.006

11. Hagberg H, Mallard C, Ferriero DM, Vannucci SJ, Levison SW, Vexler ZS, et al. The role of inflammation in perinatal brain injury. Nat Rev Neurol. (2015) 11:192-208. doi: 10.1038/nrneurol.2015.13

12. Hellstrom Erkenstam N, Smith PL, Fleiss B, Nair S, Svedin P, Wang W, et al. Temporal characterization of microglia/macrophage phenotypes in a mouse model of neonatal hypoxic-ischemic brain injury. Front Cell Neurosci. (2016) 10:286. doi: 10.3389/fncel.2016.00286

13. Xie C, Zhou K, Wang X, Blomgren K, Zhu C. Therapeutic benefits of delayed lithium administration in the neonatal rat after cerebral hypoxia-ischemia. PLoS ONE. (2014) 9:e107192. doi: 10.1371/journal.pone.0107192

14. Ziemka-Nalecz M, Jaworska J, Zalewska $T$. Insights into the neuroinflammatory responses after neonatal hypoxia-ischemia. J Neuropathol Experi Neurol. (2017) 76:644-54. doi: 10.1093/jnen/nlx046

15. Jin WN, Shi SX, Li Z, Li M, Wood K, Gonzales RJ, et al. Depletion of microglia exacerbates postischemic inflammation and brain injury. J Cereb Blood Flow Metab. (2017) 37:2224-36. doi: 10.1177/0271678X17694185

16. Li M, Li Z, Ren H, Jin WN, Wood K, Liu Q, et al. Colony stimulating factor 1 receptor inhibition eliminates microglia and attenuates brain injury after intracerebral hemorrhage. J Cereb Blood Flow Metab. (2017) 37:238395. doi: 10.1177/0271678X16666551

17. Merry TL, Brooks AES, Masson SW, Adams SE, Jaiswal JK, Jamieson SMF, et al. The CSF1 receptor inhibitor pexidartinib (PLX3397) reduces tissue macrophage levels without affecting glucose homeostasis in mice. Int J Obes. (2020) 44:245-53. doi: 10.1038/s41366-019-0355-7

18. Stafford JH, Hirai T, Deng L, Chernikova SB, Urata K, West BL, et al. Colony stimulating factor 1 receptor inhibition delays recurrence of glioblastoma after
Zhengzhou Collaborative Innovation Major Project (Grants 18XTZX12009).

\section{ACKNOWLEDGMENTS}

The authors thank Dr. Zhiguo Li for technical assistance. radiation by altering myeloid cell recruitment and polarization. Neuro Oncol (2016) 18:797-806. doi: 10.1093/neuonc/nov272

19. Alibrahim A, Zhao LY, Bae CY, Barszczyk A, Sun CL, Wang GL, et al. Neuroprotective effects of volume-regulated anion channel blocker DCPIB on neonatal hypoxic-ischemic injury. Acta Pharmacol Sin. (2013) 34:1138. doi: 10.1038/aps.2012.148

20. Rodriguez J, Xie C, Li T, Sun Y, Wang Y, Xu Y, et al. Inhibiting the interaction between apoptosis-inducing factor and cyclophilin A prevents brain injury in neonatal mice after hypoxia-ischemia. Neuropharmacology. (2020) 171:108088. doi: 10.1016/j.neuropharm.2020.108088

21. Xie C, Ginet V, Sun Y, Koike M, Zhou K, Li T, et al. Neuroprotection by selective neuronal deletion of Atg7 in neonatal brain injury. Autophagy. (2016) 12:410-23. doi: 10.1080/15548627.2015.1132134

22. Sheldon RA, Windsor C, Ferriero DM. Strain-related differences in mouse neonatal hypoxia-ischemia. Dev Neurosci. (2018) 40:490-6. doi: 10.1159/000495880

23. Zhu C, Wang X, Xu F, Bahr BA, Shibata M, Uchiyama Y, et al. The influence of age on apoptotic and other mechanisms of cell death after cerebral hypoxiaischemia. Cell Death Different. (2005) 12:162-76. doi: 10.1038/sj.cdd.4401545

24. Ye L, Feng Z, Doycheva D, Malaguit J, Dixon B, Xu N, et al. CpG-ODN exerts a neuroprotective effect via the TLR9/pAMPK signaling pathway by activation of autophagy in a neonatal HIE rat model. Experi Neurol. (2018) 301:70-80. doi: 10.1016/j.expneurol.2017.12.008

25. Yao Y, Miao W, Liu Z, Han W, Shi K, Shen Y, et al. Dimethyl fumarate and monomethyl fumarate promote post-ischemic recovery in mice. Transl Stroke Res. (2016) 7:535-47. doi: 10.1007/s12975-016-0496-0

26. Mirza MA, Ritzel R, Xu Y, McCullough LD, Liu F. Sexually dimorphic outcomes and inflammatory responses in hypoxic-ischemic encephalopathy. J Neuroinflam. (2015) 12:32. doi: 10.1186/s12974-015-0251-6

27. Tsuji S, Di Martino E, Mukai T, Tsuji S, Murakami T, Harris RA, et al Aggravated brain injury after neonatal hypoxic ischemia in microglia-depleted mice. J Neuroinflam. (2020) 17:111. doi: 10.1186/s12974-020-01792-7

28. Cengiz P, Zafer D, Chandrashekhar JH, Chanana V, Bogost J, Waldman A, et al. Developmental differences in microglia morphology and gene expression during normal brain development and in response to hypoxia-ischemia. Neurochem Int. (2019) 127:137-47. doi: 10.1016/j.neuint.2018.12.016

29. Umekawa T, Osman AM, Han W, Ikeda T, Blomgren K. Resident microglia, rather than blood-derived macrophages, contribute to the earlier and more pronounced inflammatory reaction in the immature compared with the adult hippocampus after hypoxia-ischemia. Glia. (2015) 63:222030. doi: 10.1002/glia.22887

30. Suenaga J, Hu X, Pu H, Shi Y, Hassan SH, Xu M, et al. White matter injury and microglia/macrophage polarization are strongly linked with age-related long-term deficits in neurological function after stroke. Experi Neurol. (2015) 272:109-19. doi: 10.1016/j.expneurol.2015.03.021

31. Villapol S, Faivre V, Joshi P, Moretti R, Besson VC, Charriaut-Marlangue C. Early sex differences in the immune-inflammatory responses to neonatal ischemic stroke. Int J Molr Sci. (2019) 20:3809. doi: 10.3390/ijms20153809

32. Rice RA, Spangenberg EE, Yamate-Morgan H, Lee RJ, Arora RP, Hernandez MX, et al. Elimination of microglia improves functional outcomes following extensive neuronal loss in the hippocampus. J Neurosci. (2015) 35:997789. doi: 10.1523/JNEUROSCI.0336-15.2015

33. Jeong $\mathrm{HK}$, Ji K, Min K, Joe EH. Brain inflammation and microglia: facts and misconceptions. Experi Neurobiol. (2013) 22:59-67. doi: 10.5607/en.2013.22.2.59

34. Caldeira C, Oliveira AF, Cunha C, Vaz AR, Falcão AS, Fernandes A, et al. Microglia change from a reactive to an age-like phenotype with the time in culture. Front Cell Neurosci. (2014) 8:152. doi: 10.3389/fncel.2014.00152 
35. Lambertsen KL, Clausen $\mathrm{BH}$, Babcock AA, Gregersen R, Fenger C, Nielsen $\mathrm{HH}$, et al. Microglia protect neurons against ischemia by synthesis of tumor necrosis factor. J Neurosci. (2009) 29:1319-30. doi: 10.1523/JNEUROSCI.5505-08.2009

36. Schell JB, Crane CA, Smith MF, Jr., Roberts MR. Differential ex vivo nitric oxide production by acutely isolated neonatal and adult microglia. $J$ Neuroimmunol. (2007) 189:75-87. doi: 10.1016/j.jneuroim.2007.07.004

37. Zhu C, Xu F, Wang $\mathrm{X}$, Shibata $\mathrm{M}$, Uchiyama $\mathrm{Y}$, Blomgren $\mathrm{K}$, et al. Different apoptotic mechanisms are activated in male and female brains after neonatal hypoxia-ischaemia. J Neurochem. (2006) 96:1016-27. doi: 10.1111/j.1471-4159.2005.03639.x

38. Shi K, Tian DC, Li ZG, Ducruet AF, Lawton MT, Shi FD. Global brain inflammation in stroke. Lancet Neurol. (2019) 18:1058-66. doi: 10.1016/S1474-4422(19)30078-X

39. Chhor V, Le Charpentier T, Lebon S, Ore MV, Celador IL, Josserand J, et al. Characterization of phenotype markers and neuronotoxic potential of polarised primary microglia in vitro. Brain Behav Immun. (2013) 32:7085. doi: 10.1016/j.bbi.2013.02.005

40. Liu XH, Kwon D, Schielke GP, Yang GY, Silverstein FS, Barks JD. Mice deficient in interleukin-1 converting enzyme are resistant to neonatal hypoxic-ischemic brain damage. J Cereb Blood Flow Metab. (1999) 19:1099108. doi: 10.1097/00004647-199910000-00006

41. Arvin KL, Han BH, Du Y, Lin SZ, Paul SM, Holtzman DM. Minocycline markedly protects the neonatal brain against hypoxic-ischemic injury. Ann Neurol. (2002) 52:54-61. doi: 10.1002/ana.10242

42. Hedtjarn M, Leverin AL, Eriksson K, Blomgren K, Mallard C, Hagberg H. Interleukin-18 involvement in hypoxic-ischemic brain injury. $J$ Neurosci. (2002) 22:5910-9. doi: 10.1523/JNEUROSCI.22-14-05910. 2002

43. Nazmi A, Albertsson AM, Rocha-Ferreira E, Zhang X, Vontell R, Zelco A, et al. Lymphocytes contribute to the pathophysiology of neonatal brain injury. Front Neurol. (2018) 9:159. doi: 10.3389/fneur.2018.00159
44. Li X, Aishan B, Yang Y, Xie Y, Lati D, Tuerxun P. Chemokine (C-C motif) ligand 6 aggravates hypoxia reoxygenation-induced apoptosis in $\mathrm{H} 9 \mathrm{c} 2$ cells through enhancing the expression of insulinlike growth factor 2-antisense. J Cardiovasc Pharmacol. (2020) 76:549-55. doi: 10.1097/FJC.0000000000000905

45. Smith AM, Gibbons HM, Oldfield RL, Bergin PM, Mee EW, Curtis MA, et al. M-CSF increases proliferation and phagocytosis while modulating receptor and transcription factor expression in adult human microglia. $J$ Neuroinflamm. (2013) 10:85. doi: 10.1186/1742-2094-10-85

46. Liu W, Brosnan CF, Dickson DW, Lee SC. Macrophage colony-stimulating factor mediates astrocyte-induced microglial ramification in human fetal central nervous system culture. Am J Pathol. (1994) 145:48-53.

47. Lacey DC, Achuthan A, Fleetwood AJ, Dinh H, Roiniotis J, Scholz GM, et al. Defining GM-CSF- and macrophage-CSF-dependent macrophage responses by in vitro models. J Immunol. (2012) 188:5752-65. doi: 10.4049/jimmunol.1103426

48. Verreck FA, de Boer T, Langenberg DM, Hoeve MA, Kramer M, Vaisberg E, et al. Human IL-23-producing type 1 macrophages promote but IL-10producing type 2 macrophages subvert immunity to (myco)bacteria. Proc Natl Acad Sci USA. (2004) 101:4560-5. doi: 10.1073/pnas.0400983101

Conflict of Interest: The authors declare that the research was conducted in the absence of any commercial or financial relationships that could be construed as a potential conflict of interest.

Copyright (c) 2021 Zhang, Ran, Wu, Zhang, Huang, Zhu, Zhang and Zhang. This is an open-access article distributed under the terms of the Creative Commons Attribution License (CC BY). The use, distribution or reproduction in other forums is permitted, provided the original author(s) and the copyright owner(s) are credited and that the original publication in this journal is cited, in accordance with accepted academic practice. No use, distribution or reproduction is permitted which does not comply with these terms. 